\title{
Health information technology in US emergency departments
}

\author{
Daniel J. Pallin • Ashley F. Sullivan • Rainu Kaushal • \\ Carlos A. Camargo Jr.
}

Received: 7 December 2009 / Accepted: 10 February 2010/Published online: 1 April 2010

(C) Springer-Verlag London Ltd 2010

\begin{abstract}
Background Information technology may improve patient safety, and is a focus of health care reform. A minority of emergency departments (EDs) in Massachusetts, and in academic EDs throughout the US, have electronic health records.

Aims Assess health information technology adoption in a nationwide sample of EDs.

Methods We surveyed 69 US EDs, asking site investigators about the availability of health information technology in 2005-2006. Using multiple linear regression, we compared adoption of technology by ED type (emergency medicine
\end{abstract}

Funding This project was supported by grant no. 5 R01 HS013099 from the Agency for Healthcare Research and Quality (Rockville, MD).

D. J. Pallin $(\bowtie)$

Department of Emergency Medicine,

Brigham \& Women's Hospital,

75 Francis St.,

Boston, MA 02115, USA

e-mail: dpallin@partners.org

D. J. Pallin

Division of Emergency Medicine, Children's Hospital Boston, Boston, MA, USA

A. F. Sullivan · C. A. Camargo Jr. Department of Emergency Medicine,

Massachusetts General Hospital,

Boston, MA, USA

C. A. Camargo Jr.

Department of Medicine, Massachusetts General Hospital,

Boston, MA, USA

R. Kaushal

Departments of Pediatrics, Medicine and Public Health,

Weill Cornell Medical College,

New York, NY, USA residency affiliation, annual census, US region) to assess generalizability of the findings.

Results Sixty-eight EDs (99\%) provided information about health information technology; $75 \%$ were affiliated with an emergency medicine residency, and all were urban. Most respondents had applications that simply relay information from one place to another, including patient tracking (74\%); ordering tests (laboratory 57\%, others $62 \%$ ); and displaying prior visit notes (79\%), ECGs (92\%), laboratory (97\%), and radiology (99\%) results. A minority had more-advanced applications, which seek to modify human behavior, including medication ordering $(38 \%)$, allergy warnings $(19 \%)$, and medication crossreaction warnings $(13 \%)$, and a few used bar coding (20\%). There were no significant differences in technology adoption by ED type.

Conclusions This and prior studies suggest that some applications-particularly those relevant to modifying clinician behavior - are not widespread in US EDs, while others are. The reasons for this are unknown, but might include expense and unintended consequences. The fact that the emergency medicine community has not rushed to adopt certain applications presents challenges and opportunities.

Keywords Information technology Electronic health records $\cdot$ Emergency department $\cdot$ Patient safety

\section{Introduction}

Health information technology has the potential to improve health care. In early 2009, the American Reinvestment and Recovery Act allocated $\$ 19$ billion for health information technology funding, and Medicare and Medicaid offer 
incentives for "meaningful use" of electronic medical records [1]. However, cost, difficulty of implementation, and other barriers impede adoption of such systems, and studies have documented low rates of technology acquisition and implementation in emergency department (ED) and other settings [2].

A study of residency-affiliated EDs in 2000 found low rates of adoption, with only $7 \%$ reporting fully implemented technology for medication error checking, $18 \%$ for computerized medication order entry, and $21 \%$ for clinical documentation. A 2006 survey of health information technology in all Massachusetts EDs found similar results, with $11 \%$ of respondents reporting fully implemented technology for medication error checking [2]. Another national study showed that, during 2001-2003, only 31\% of US EDs used electronic medical records in any form [3].

Our hypothesis is that fewer than half of US EDs had adopted electronic health records systems and related decision-support tools by 2005-2006.

\section{Methods}

We report on data collected as part of the National ED Safety Study (NEDSS). Details of the study design and data collection have been published previously [4]. NEDSS was coordinated by the Emergency Medicine Network (EMNet, www.emnet-usa.org). We recruited EDs mainly by inviting network members. Since most members are affiliated with an emergency medicine residency program (i.e., are academic EDs), we also recruited non-academic and other EDs not affiliated with EMNet through postings on emergency medicine list servers, by contacting sites directly, and through presentations at emergency medicine meetings. We excluded federal hospitals, children's hospitals, and hospitals in US territories.

For the parent National ED Safety Study, each site had a Site Responsible Investigator. For the present study, that person provided information by filling out a survey. The survey asked about ED attributes and adoption of the health information technology applications shown in Table 1. These applications were chosen by the authors as representative of the diverse applications available today, within the constraints of a larger survey with other goals. We performed all calculations with SAS 9.12 (SAS Institute, Cary, NC). The Institutional Review Board of each participating hospital approved the study.

Our main outcome measure is descriptive: how many EDs have adopted which applications?

Our secondary objective was to determine whether ED characteristics predicted adoption of health information technology. We created two methods for addressing this question.
Table 1 Characteristics of 68 emergency departments surveyed ${ }^{\mathrm{a}}$

\begin{tabular}{lll}
\hline Characteristic & N (\%) & $\begin{array}{l}\text { Median computerization } \\
\text { score }^{\mathrm{b}}\end{array}$ \\
\hline $\begin{array}{l}\text { Emergency medicine residency affiliation } \\
\text { Yes }\end{array}$ & $51(75 \%)$ & 9 \\
No & $17(25 \%)$ & 8 \\
Annual census (visit per year) & \\
$<10,000$ & 0 & $\mathrm{n} / \mathrm{a}$ \\
$10,000-19,999$ & 0 & $\mathrm{n} / \mathrm{a}$ \\
$20,000-29,999$ & $1(1 \%)$ & 5 \\
$30,000-39,999$ & $12(18 \%)$ & 8.5 \\
$40,000-49,999$ & $12(18 \%)$ & 7.5 \\
$\geq 50,000$ & $43(63 \%)$ & 9 \\
US region & & 8 \\
Northeast & $30(44 \%)$ & 9 \\
Midwest & $15(22 \%)$ & 9 \\
South & $10(15 \%)$ & 8 \\
West & $13(19 \%)$ & \\
\hline
\end{tabular}

${ }^{a}$ We surveyed key informants at 69 US EDs, as part of the National Emergency Department Safety Study [4], with 68 (99\%) of EDs surveyed responding

${ }^{\mathrm{b}}$ The Computerization Score was simply a way for us to count the number of information technologies that had been acquired. We calculated the score by assigning one point for each acquired application listed in Table 2

Firstly, we selected one application that seemed to represent the potential of information technology to improve health care. There is substantial debate about what constitutes "meaningful use" of information technology, and we felt that selecting one crucial application for analysis would be the best way to compare across EDs. For the purpose of this analysis, we selected computerized medication ordering as the application. We chose this application because medication ordering represents a crucial site of potential intervention to improve adherence to recommended care and avoid error. We studied this as an outcome variable by creating a logistic regression model with computerized medication order entry as the dependent variable. We then used the model to determine whether ED characteristics predicted its adoption. We categorized EDs according to emergency medicine residency affiliation (binary), annual census (continuous), and region (categorical: Northeast, Midwest, South, and West). We used multiple logistic regression to model these characteristics as predictors of adoption of computerized medication ordering. We used the Hosmer-Lemeshow goodness of fit test to evaluate for non-linearity.

Secondly, we did not wish to limit our investigation to any one application or combination of applications, as was done in the analysis described above. Thus, we also compared EDs by the raw number of applications they 
had adopted. We accomplished this by creating a computerization score, as was done in a prior study [5]. This score is simply a count of applications, with one point assigned for each acquired application. We then used multiple linear regression to predict computerization score by the above ED characteristics and planned to consider any characteristic to be a "significant" predictor of the computerization score if the p-value of its beta coefficient was $\leq 0.05$. We used the SAS spec option to assess for heteroscedasticity and inspected residual plots for qualitative evidence of a relationship.

\section{Results}

Of 69 EDs surveyed, 68 (99\%) in 23 US states completed the survey. Table 1 shows the characteristics of EDs surveyed. The participants were generally large urban academic EDs, with $44 \%$ located in the Northeast.

Table 2 shows the number and proportion of EDs reporting availability of selected health information technologies. Computerized physician order entry, clinical decision support, and bar coding technology were present in fewer than $40 \%$ of EDs. In contrast, some other applications were present in most of the EDs surveyed, including laboratory test ordering and results, notes from prior encounters, and ECG results. The right-hand column of Table 1 shows the computerization score (see Methods), stratified by ED characteristics.

Our analysis of ED characteristics did not reveal any significant variation in availability of electronic medication ordering or computerization score, by emergency medicine residency affiliation, annual census, or region.

\section{Discussion}

Information technology is ubiquitous in our society. Businesses from restaurants to banks and airlines rely on such technology to process information that has much in common with medical information. Such technology has been recommended strongly for health care settings [1]. Why, then, would our nationwide sample of EDs reveal that only $19 \%$ have the capability to check a medication order to see if the patient has a documented allergy to that medication?

Reasons might include apprehension. In the enthusiasm to promulgate the spread of health information technology, the downsides are often under-emphasized [6]. Moreover, purchase and implementation of such technology is not necessarily cost-effective [7].

The present report echoes the findings of the three prior studies of health information technology adoption in US EDs - namely, slow uptake of certain applications [2, 3]. Only two of these prior studies assessed the adoption of individual applications, such as computerized physician order entry. One was a survey of US emergency medicine residency-affiliated EDs in 2000, and the second was a survey of all Massachusetts EDs in 2006 [2]. Our ability to conduct direct quantitative comparisons across time is limited, because different EDs participated in the surveys. However, certain qualitative observations seem valid. All three studies revealed that a minority of EDs had computerized medication ordering: $38 \%$ in the present national study, $15 \%$ in the 2006 MA study, and $18 \%$ in the 2000 national study. Quantitatively, the increase from $18 \%$ to $38 \%$ might indicate wider dissemination of these technologies, or might be due to sampling variation.
Table 2 Information technology in 68 US emergency departments
Information technology application

Number (\%) of EDs reporting availability of each application

\begin{tabular}{ll}
\hline Computerized physician medication order entry & $26(38 \%)$ \\
Clinical decision support for medication allergies & $13(19 \%)$ \\
Clinical decision support for drug-drug interactions & $9(13 \%)$ \\
Outpatient notes & $39(57 \%)$ \\
Inpatient notes & $43(63 \%)$ \\
Surgical notes & $53(78 \%)$ \\
Prior ED visit notes & $54(79 \%)$ \\
Laboratory results & $66(97 \%)$ \\
Laboratory test ordering & $39(57 \%)$ \\
Test ordering other than laboratory tests & $42(62 \%)$ \\
Radiology results & $67(99 \%)$ \\
EKG results & $62(92 \%)$ \\
Patient tracking & $50(74 \%)$ \\
Bar coding for patient, medication, or sample identification & $14(20 \%)$
\end{tabular}


Qualitatively, it seems reasonable to conclude from these three studies that most US EDs do not have electronic medication ordering, or its companion technology, medication error checking. However, this conclusion must be caveated by the fact that large academic EDs were overrepresented in these samples.

Studies of health information technology adoption in physicians' offices and hospitals throughout the country also revealed slow uptake by $2008[8,9]$. These studies found that only $7.6 \%$ of US hospitals have a basic electronic records system, and $1.5 \%$ a comprehensive one. (These studies defined "basic" as including only demographic information, CPOE, laboratory and imaging results, and "comprehensive" as including the above, plus clinical notes, plus electronic prescribing, radiographic image display, and decision support.) Computerized provider order entry for medications has been implemented in only $17 \%$ of hospitals. Only $4 \%$ of physicians' offices have a fully functional electronic-records system, and $13 \%$ have a basic system.

ED-based studies also show that some health information technology applications are widespread, including patient tracking; ordering tests; and displaying prior visit notes, ECGs, and laboratory and radiology results $[2,3,5]$. An analysis of the types of health information technology may improve our understanding of these results. One type of application is computationally simple, merely entering and extracting information from a database, and displaying it. The other type of application requires all of the above processes, plus algorithmic processing that seeks to supplement human cognition with computation. For lack of better terms, we might refer to these two types of application as "passive" and "active" applications, respectively. From a workflow standpoint, passive applications simply convert traditional paper processes into an electronic format. Such technologies include patient tracking; ordering tests; and displaying prior visit notes, ECGs, laboratory and radiology results. Active applications add functions designed to improve outcomes by reducing error or increasing adherence to the standard of care - they seek to change human behavior. These applications often embody fundamental changes in clinical processes, and implementation is intertwined with changes in workflow $[6,10]$. Examples include computerized provider order entry and related error-checking functions, and decision support systems. In light of this dichotomization, we can see that the present and prior studies reveal a high rate of adoption of passive applications in US EDs and a low rate of adoption of active applications [2, 3]. (We included laboratory test ordering in the "passive" category for historical reasons. Such systems have been present in most EDs for many years [5] and were not originally subject to behavior-modification technology.)
An optimistic interpretation of our data would be to celebrate the fact that most US EDs did not invest in expensive, cumbersome, and even potentially risky systems prematurely. Now that a large number of commercial and home-grown systems are available, we should take the time to study them and reach consensus on which would be best for particular environments. We should pay particular attention to the interaction of information systems with human workflows $[6,10]$. The Certification Commission for Healthcare Information Technology formed an ED Work Group in 2007, which is in the process of certifying various health information technology platforms for ED use, in concert with the Health Level Seven project [11]. This type of certification process may be an important first step in improving the types of systems that are available, or at least our understanding of what is available.

Our study is limited by the convenience sample of 68 EDs across the US. Participating sites were generally large, urban, academic EDs. Because the underlying patient safety study focused on adult illnesses, the study excluded children's hospitals. We did assess for variability in technology adoption by ED characteristics, and found no significant variation. This improves our confidence in the generalizability of our data to other EDs in the US, though, again, large academic EDs were over-represented in the sample. Perhaps more saliently, the present results are similar to those of prior studies, from academic EDs throughout the US, and all EDs in Massachusetts [2]. It remains true that we have little data on health information technology adoption in the $1 / 3$ of US EDs with an annual visit volume $<10,000$. From the perspective of the individual patient, small EDs have the same clinical goals as large EDs. But from a systems perspective, the marginal cost of health information technology in low-volume settings will be high relative to benefit, both financially and in terms of workflow modification and associated risks [8].

Another limitation is the fact that our assessment was part of a larger survey, and thus, we could not conduct as detailed an inquiry as would have been desirable. In particular, we did not ascertain adoption of health information technology for medication order checking or decision support. However, the low rate of adoption of medication order entry technology allows us to conclude with confidence that these technologies were not available in the majority of respondent EDs.

Active applications involve intertwining of technology and human workflows, and are intended to change behavior. Passive applications involve simple data processing and display. Together with the findings of other studies, our results demonstrate that most US EDs have adopted passive applications but not active applications that have been recommended forcefully $[2,3]$. We view 
this as an opportunity, because careful analysis of available platforms and the pitfalls in their implementation may result in better outcomes and less difficulty in the implementation process. The ideal forum for such analysis remains unclear, though the Certification Commission for Healthcare Information Technology ED work group is a notable effort [11].

\section{References}

1. Blumenthal D (2009) Stimulating the adoption of health information technology. N Engl J Med 360(15):1477-1479

2. Pallin DJ, Sullivan AF, Auerbach BS, Camargo CA, Jr. Adoption of Information Technology in Massachusetts Emergency Departments. J Emerg Med. 2009 Feb 18 [Epub ahead of print.] 2009

3. Burt CW, Hing E. Use of computerized clinical support systems in medical settings: United States, 2001-03. Adv Data. Mar 22005 (353): $1-8$
4. Sullivan AF, Camargo CA Jr, Cleary PD et al (2007) The national emergency department safety study: study rationale and design. Acad Emerg Med 14(12):1182-1189

5. Pallin D, Lahman M, Baumlin K (2003) Information technology in emergency medicine residency-affiliated emergency departments. Acad Emerg Med 10(8):848-852

6. Ash JS, Sittig DF, Campbell EM, Guappone KP, Dykstra RH. Some unintended consequences of clinical decision support systems. AMIA Annu Symp Proc. 2007:26-30

7. Himmelstein DU, Wright A, Woolhandler S. Hospital Computing and the Costs and Quality of Care: A National Study. Am J Med. Nov 162009

8. DesRoches CM, Campbell EG, Rao SR et al (2008) Electronic health records in ambulatory care - a national survey of physicians. N Engl J Med 359(1):50-60

9. Jha AK, DesRoches CM, Campbell EG et al (2009) Use of electronic health records in US hospitals. N Engl J Med 360 (16): $1628-1638$

10. Han YY, Carcillo JA, Venkataraman ST et al (2005) Unexpected increased mortality after implementation of a commercially sold computerized physician order entry system. Pediatrics 116 (6):1506-1512

11. http://www.cchit.org/emergency/ Accessed 3/9/09 